\title{
Quaderni
}

QUADERN I Communication, technologies, pouvoir

76 | Automne 2011

Les promesses de la biodiversité

\section{Soma ou novlangue ? À propos des nouveaux mots du pouvoir}

\section{Pascal Durand}

\section{(2) OpenEdition}

Journals

Édition électronique

URL : http://journals.openedition.org/quaderni/124

DOI : 10.4000/quaderni.124

ISSN : 2105-2956

Éditeur

Les éditions de la Maison des sciences de l'Homme

Édition imprimée

Date de publication : 1 septembre 2011

Pagination : 91-100

Référence électronique

Pascal Durand, «Soma ou novlangue? À propos des nouveaux mots du pouvoir », Quaderni [En ligne], 76 | Automne 2011, mis en ligne le 01 septembre 2014, consulté le 20 avril 2019. URL : http://

journals.openedition.org/quaderni/124; DOI : 10.4000/quaderni.124 


\section{Politique}

SOMa OU «Les mots ont une grande importance; icchapper au mot, c'est déjà entrer dans la voie de la novlangue? $\begin{aligned} & \text { liberation. } \\ & \text { Remy de Gourmont }\end{aligned}$

À propos des nouveaux mots

du pouvoir

" [Le public] écoute les bateleurs de la TV, sans se douter que dans ce déluge de mots mal employés se cachent ceux qui lui couperont la langue un jour. »

Julien Green ${ }^{2}$

En mars 2006, en temps de crise sociale autour du projet de $\mathrm{CPE}^{3}$, l'auteur d'une carte blanche sur «Les trois conditions de toute réforme", parue dans un grand quotidien belge, résumait ainsi son $\operatorname{propos}^{4}$ :

«Dans ces conditions, la réforme demeure-t-elle

\section{Pascal Durand}

Département des arts et sciences de la communication

Université de Liège possible? En l'absence d'une pédagogie de la gouvernance et de l'économie, d'une convergence des formations politiques autour des réformes essentielles et d'une restauration de l'autorité du politique, il est permis d'en douter. »

D'où vient la sensation pâteuse, l'impression d'engluement produite par une telle péroraison? Elle ne vient pas du contenu de pensée des trois propositions que celle-ci rassemble ; elle vient au contraire de ce que la teneur de cette envolée finale se résorbe tout entière, par une sorte d'implosion ou d'affaissement du propos sur lui-même, en une ponctuation de mots clichés : réforme, pédagogie, gouvernance, le politique, réformes essentielles. Comme tant d'autres, l'auteur de cette tribune est persuadé - et entend bien persuader son lecteur - non seulement de l'importance et de l'intelligence de ce qu'il énonce avec une grande hauteur de vue apparente, mais 
encore qu'il pense contre l'opinion commune, les inerties collectives, l'immobilisme ambiant. Et pourtant tout se passe dans ces quelques lignes comme si les éléments constitutifs du discours avaient pensé à la place de celui qui s'est employé très spontanément à les disposer sous nos yeux. Regardons-y mieux en effet : de tels clichés et surtout ces clichés-là : réforme, pédagogie, gouvernance, le politique - ne sont pas venus par hasard sous la plume de notre auteur, ni sous l'impulsion tout intérieure d'une conviction fermement délibérée. Ces clichés appartiennent à un répertoire assez restreint de mots et d'expressions qui sont parmi les plus récurrents, depuis une quinzaine d'années, dans le discours social et politique; des mots et des expressions qui sont à la pensée ce que le chewing-gum est à la nutrition : une mastication qui tournerait à vide si elle ne contribuait pas au conditionnement général des esprits du côté d'une représentation libérale du monde qui se pense - et se donne les moyens d'être pensée et acceptée - comme le nouvel horizon indépassable de nos sociétés post-industrielles.

Il y a pourtant une pensée à l'œuvre dans cette mastication, et très cohérente, sans qu'il y ait besoin de l'attribuer à l'auteur de cette carte blanche, lequel ne fait ici, sans trop le savoir, que réciter une sorte de credo bien propre à lui valoir le brevet de modernité requis pour accéder à ce qu'il est convenu d'appeler, depuis qu'un Alain Minc l'a baptisé ainsi, le cercle de la raison. Quelle est cette pensée?

D'abord l'idée, impliquée par le vocable de pédagogie si régulièrement attelé à celui de réformes, d'une société composée, au sommet, d'une élite d'esprits clairvoyants autant que bienveillants et, par ailleurs, d'une population vaguement puérile, peu ou mal informée, rebelle, capricieuse, n'ayant pas encore intégré le principe de réalité et qui, lorsqu'elle se rebiffe, ne le fait que dans la mesure où elle n'a pas compris qu'on veut son bien. Pédagogie de quoi, ensuite? «Pédagogie de la gouvernance et de l'économie », c'est-àdire, d'un côté, d'une direction expertocratique de la société, chevillée à une représentation de la politique et du gouvernement comme gestion techno-économique de ressources matérielles et humaines, et, de l'autre, pédagogie d'une économie pensée non comme diversité de choix ou de stratégies possibles mais comme ensemble de lois auxquelles il n'y aurait pas d'alternative. Proposition d'une très grande circularité : car au fond gouvernance et pédagogie s'impliquent mutuellement. La politique vue comme affaire d'experts, l'économie vue comme nature répondant à des lois d'airain supposent une irréductible inégalité entre ceux qui savent et ceux qui ne savent pas : d'une part, des techniciens de la chose publique, humblement avertis des complexités du monde; d'autre part, une masse nombreuse mais en situation de minorité intellectuelle, parfois rétive, bien inutilement, à l'ordre du monde, mais parce qu'elle est peu armée pour en comprendre les arcanes et qu'elle est par conséquent la proie toute désignée des instincts primaires, des replis frileux et des slogans simplistes. ${ }^{5}$

Deuxième proposition, deuxième condition à « toute réforme» pour notre auteur : la « convergence des formations politiques autour des réformes essentielles ». La machine tourne en rond, mais pas forcément à vide. Voici effectivement que l'adhésion unanime à des « réformes essen- 
tielles » conditionne « la réforme ». Comprenons dès lors qu'il y a d'un côté des réformes locales, pratiques, des ajustements ou des remises en question nécessaires pour que, de l'autre, une réforme générale de la société soit enfin mise en œuvre. Et voici la réforme instituée non plus seulement en moyen, mais en objectif et en enjeu (ce qui explique les sauts de cabri à son sujet de la plupart des hommes et femmes de gouvernement, dans certaines instances syndicales ou dans les organisations patronales). La réforme serait, au fond, quelque chose comme le Graal de nos modernes chevaliers de l'économie et de la politique (après tout, les industriels européens ont aussi leur table ronde). L'idée apparemment si séduisante aux yeux de notre auteur d'une " convergence des formations politiques » s'ordonne assez bien, elle aussi, à ce que la gouvernance implique : une gestion rationnelle de la société avec, dans l'absolu, une politique dépolitisée, une politique enfin soustraite au conflit, aux rapports de force, et, plus concrètement, ajustée à un esprit de consensus favorisant à terme l'alternance, au pouvoir, de deux formations d'accord sur l'essentiel - et d'ores et déjà sur les « réformes nécessaires 》-, formations qui ne seraient plus guère en désaccord que sur des aspects marginaux de la politique à conduire et de la stratégie politique à mener pour y parvenir.

Troisième proposition, troisième condition de « la réforme » : la « restauration de l'autorité du politique ». Notons, en passant, politique au masculin. Le politique, c'est plus chic désormais que $l a$ politique. La politique, ça sent la sueur des meetings, ça évoque le bras de fer, le coup de force, les petites stratégies minables autant que les grands mouvements d'opinion, et la rhétorique des tribuns plus que l'éloquence policée des politologues. Le politique donc, mais ici encore comme instance située au-delà de la politique au-delà de la gauche et de la droite, comme on serait au-delà du bien et du mal, en tout cas dans cette région apaisée où règnera enfin un consensus conforme à la raison et adapté à un monde de plus en plus complexe. Reste enfin cette «restauration de l'autorité du politique », par quoi d'un côté notre auteur échappe à l'assignation soupçonneuse à une pensée du tout-au-marché; il y a place, dans sa pensée, pour l'État, c'est-àdire pour une pensée de l'État vu lui aussi comme instance en surplomb ou bien vu, si l'on est de droite, comme garant de l'ordre public et, si l'on est de la gauche dite altermondialiste, comme pure garantie de l'intérêt public. Mais par quoi aussi, d'un autre côté, notre auteur n'échappe pas, cependant, au risque de recycler le vieux cliché réactionnaire du "déclin de l'autorité », de la même façon qu'il recyclait à son insu, avec le motif de la pédagogie l'une des figures classiques de la rhétorique réactionnaire du $\mathrm{XIX}^{\mathrm{e}}$ siècle dans sa variante adoucie, lorsque le bourgeois paternaliste installait face à lui l'image d'un peuple enfant. ${ }^{6}$

Lire d'aussi près un tel texte de circonstance, c'est sans doute lui faire plus d'honneur que l'auteur lui-même n'en attendait de son lecteur. Mais il s'agissait ici de faire ressortir deux ou trois choses : d'abord l'extrême banalité d'un propos entendu cent fois et débité à longueur de colonnes et de déclarations d'intention par les éditorialistes de référence, les gens de pouvoir et autres décideurs; ensuite, la grande circularité de ce propos, un propos que sa forme tautologique range, mieux encore que son contenu, du côté du discours de l'idéologie en général, toutes 
sensibilités confondues; enfin, la cohérence de la vision du monde qu'elle met au service de la nécessaire « réforme » de nos sociétés.

Mais venons-en aux mots du pouvoir et, plus précisément, à ces "nouveaux mots du pouvoir » dont toute une équipe de chercheurs a établi l'abécédaire critique dans un copieux volume paru sous ce titre : soit près de cent cinquante mots en trafic constant dans le discours politique et social depuis une quinzaine d'années environ. ${ }^{7}$ Les trois propositions qui viennent d'être examinées en présentaient quelques-uns, parmi ceux dotés du plus haut taux de fréquence : gouvernance, réforme, pédagogie (ou explication). En voici quelques autres en vrac : flexibilité, dialogue social, partenaires sociaux, modernité, excellence, performance, restructurations, adaptation, changement, développement durable, Europe, contraintes structurelles, compétitivité, employabilité, échec du référendum, gauche de la gauche, défi, mondialisation, déficit de communication, etc. Un peu de sens combinatoire suffirait, sans grand effort, pour composer avec eux des éditoriaux à la chaîne, des déclarations gouvernementales en série, pour jouer sans peine dans la cour de François Fillon et de Laurence Parisot, de Manuel Valls et de Bertrand Delanoé ou dans celle, pour la Belgique, du libéral Alain Destexhe et du premier ministre social-chrétien flamand Yves Leterme, comme aussi bien du socialiste Rudy Demotte et de la social-chrétienne wallonne Joëlle Milquet (présidente du $\mathrm{CDH}^{8}$ ).

Car ces mots traversent les compartimentations politiques et sont peut-être bien l'opérateur verbal de cette « convergence des formations politiques » qu'appelait si ardemment de ses vœux l'auteur de notre carte blanche.

Est-ce là un phénomène nouveau ? Oui et non.

Ce n'est pas un phénomène nouveau, parce que, nous le savons bien, la politique est affaire de discours autant que d'action, et le pouvoir en particulier ne se conçoit pas sans une certaine autorité exercée par le langage et sur le langage. Autorité qui consiste au fond non seulement à imposer des mots, mais à circonscrire avec eux le champ du dicible et du pensable, à faire sentir non seulement comment les choses doivent être dites, mais quelles choses sont susceptibles d'être dites et à quel principe de pertinence elles sont ordonnées dans l'ordre du discours. Les mots n'ont pas de pouvoir intrinsèque, mais le pouvoir passe par des mots, s'exerce et se maintient comme force de conformité à travers un conditionnement des esprits qui passe par les mots bien plus au fond que par des arguments. Et ce pouvoir à travers les mots ne s'exerce jamais aussi bien que lorsque ces mots sortent de leur sphère politique d'appartenance pour circuler très largement, comme on le voit notamment avec ceux qui nous occupent, à travers les médias, la publicité, les conversations de comptoir ; ou bien, mieux encore, lorsque les mots de l'économie tendent, comme on le voit depuis quelques années, à s'appliquer à des domaines qui ne relèvent pas de l'économie ou qui, jusqu'ici, n'en relevaient pas de plein fait (l'école ou l'université par exemple, les rapports sociaux, les rapports amoureux, etc.).

Il y a, cependant, du neuf dans ces mots pris ici pour cibles et pour témoins du discours social et politique dans lequel nous sommes actuellement plongés. Nouveauté d'abord qui est celle de leur capacité à transcender les clivages politiques. 
Jadis la gauche et la droite avaient leurs mots, s'affrontaient à travers des lexiques contrastés ; aujourd'hui ces frontières lexicales ont cédé et, de la droite décomplexée à la gauche d'exécution, ce sont en gros les mêmes mots qui sont en usage, au prix en certains cas très significatifs d'une translation de mots d'un camp politique vers l'autre : ainsi du mot de réforme, passé de la gauche réformiste à la droite libérale, avant d'être repris en charge par la gauche dite moderne ou gestionnaire. C'est dans le même sens que les mots de conservateur et de progressiste ont troqué leurs places sur l'échiquier politique. Rien ne montre mieux sans doute le pouvoir du discours libéral que sa capacité à incorporer les mots du camp qui lui était opposé et, dans le même mouvement, à imposer ses mots et la conception du monde qui les enveloppe au discours de gauche censé s'opposer à lui. Parler avec les mots de l'adversaire, c'est avoir déjà rendu les armes. Ainsi, lorsque le socialiste Rudy Demotte, au moment de prendre ses fonctions de ministre président de la Communauté française de Belgique, déclare dans sa première intervention dans la presse qu'il entend «libérer les forces du marché », c'est autant par les mots employés ( « libérer », « forces », « forces du marché ») que par le contenu de son projet qu'il exprime, non peut-être son ralliement, mais son consentement à l'ordre libéral dominant.

C'est là, soit dit entre parenthèses, pourquoi ces mots et ces discours ne relèvent que partiellement de ce qu'il est convenu, depuis Orwell, d'appeler la novlangue. De cette novlangue, ils ont bien sûr la capacité sédative, son pouvoir d'appauvrissement de la langue et d'endoctrinement de la pensée, son pouvoir aussi de destruction du sens logique. Mais cette novlangue, Orwell l'a pensée en fonction d'un modèle totalitaire qui n'est plus le nôtre ; notre totalitarisme n'est plus un totalitarisme fasciste ou stalinien ; c'est aujourd'hui le modèle libéral qui s'est imposé comme forme dominante et comme vision totalisante du monde ; totalitarisme, si l'on veut, mais bien difficile à circonscrire et à rendre sensible puisqu'il s'exprime à travers des valeurs affichées de liberté, à travers une prescription d'autonomie du sujet et l'injonction paradoxale d'un affranchissement à l'égard de toute règle collectivement délibérée. Ce n'est plus l'asservissement qui est prescrit au sujet : c'est sa libération; ce n'est plus l'immobilité, le conservatisme : ce sont le mouvement, le changement, l'évolution; ce n'est plus le renoncement, l'abnégation, mais au contraire la pleine réalisation de ses potentialités et de ses désirs. Si bien que s'il faut assigner pour horizon rétrospectif à notre discours et à nos idéologies d'aujourd'hui une utopie du passé, plutôt qu'au roman d'Orwell, c'est au Meilleur des mondes (1932) d'Aldous Huxley qu'il conviendrait de s'adresser, évocation glaçante d'une société de castes, avec ses élites et ses ilotes ; malthusienne et biotechnologique ; soumise à hypnopédie, à constante persuasion euphorisante, au déni de l'histoire et à un utilitarisme généralisé, mais combiné avec le règne impératif de l'entertainment. Une société dans laquelle la prise régulière d'une petite pilule, le soma, garantit l'euphorique adaptation du sujet à son environnement.

Ce qu'il y a de neuf, d'autre part, dans l'ensemble de ce lexique, c'est qu'il s'articule étroitement à une nouvelle représentation à la fois très univoque et diffuse du monde social et politique. Gouvernance, compétitivité, flexibilité, rationalisation, 
dialogue social, etc. : ces nouveaux mots du discours politique ordinaire, parmi tant d'autres, répondent bien moins à des réalités nouvelles, qu'il s'agirait de nommer, qu'à une nouvelle construction de la réalité politique et sociale. L'idée qu'il y a des réalités nouvelles qu'il faut affronter, que les choses deviennent de plus en plus complexes, etc. est l'un des leitmotiv les plus irritants du discours actuel du pouvoir. Sociétal à la place de « social », gouvernance à la place de «politique» ou de « gouvernement», le politique à la place de «la politique » correspondent, tout en y contribuant, à un façonnement particulier de cette réalité politique et sociale. Le mot le plus significatif, autour duquel tous les autres tendent à rayonner, est bien entendu celui de gouvernance : emprunté à l'anglais du management (la corporate governance), il réduit la politique à une technologie de gestion, à une administration de ressources (la société est vue, en somme, comme une méga-entreprise et l'entreprise ellemême comme le site d'élevage d'un nouveau type anthropologique, vaguement schizoïde, hésitant entre l'individu calculateur et l'employé précaire, machine performante et flexible dans la sphère de la production, mais machine désirante dans la sphère de la consommation ${ }^{9}$ ); il place le pouvoir de choix et de décision entre les mains d'une expertocratie tout en élargissant la sphère de la décision politique à des acteurs qui, jusqu'ici, relevaient du privé, qu'il s'agisse de ce qu'on appelle les « acteurs économiques », sinon même les représentants de différentes confessions religieuses ; et de la même façon il tend simultanément à élargir le régime des stratégies économiques au domaine des choix politiques et, tout en faisant fond sur une vision compétitionnelle de la société, à lisser les contradictions, les conflits, à occulter, sinon à dissoudre, la dialectique sociale qui est au principe du débat et de l'action politiques en démocratie. Des expressions si reçues désormais comme celles de dialogue social ou de partenaires sociaux font d'évidence système avec ce principe de « gouvernance » pour entériner l'idée d'une fin de l'Histoire qui serait aussi une fin de la politique (c'est d'ailleurs cette fin de la politique que le politique nomme aujourd'hui en règle générale).

Guy Hermet soutient, dans L'hiver de la démocratie, que sans trop nous en rendre compte nous serions entrés dans un régime post-démocratique ; la gouvernance serait ainsi aux élites ce que le populisme est aux classes dominées : un déni et un mépris de la politique, une dévitalisation de la démocratie, de plus en plus formelle, de moins en moins vécue comme mise en conflit de projets de société et comme contrôle exercé par le peuple sur ses représentants. ${ }^{10}$ « La polis grecque, écrivait déjà Hannah Arendt, continuera d'être présente au fondement de notre existence politique [...] aussi longtemps que nous aurons à la bouche le mot "politique" " ${ }^{12}$. Avec gouvernance, qui tend à s'y substituer, le moment semble bien venu de l'oubli de cette polis et du modèle démocratique dont elle est la forme la plus archaïque. Derrière l'expression de dialogue social ou de partenaires sociaux, on peut au mieux entrevoir le lissage des conflits, et au pire l'alliance du Capital et du Travail qu'un Fritz Lang avait mis en scène à la fin de Metropolis (1936). Et en ce sens il est sans doute anachronique aujourd'hui de penser que nos sociétés se trouvent soumises à ce qu'il est convenu d'appeler une politique néo-libérale ; cette impulsion néo-libérale est derrière nous, elle a rempli son office historique et nos sociétés sont 
bien plutôt en train de passer sous le régime d'une néo-politique - pour reprendre une expression de Pierre Musso -, c'est-à-dire une politique articulant management et communication, État autoritaire et marché dérégulé. De cette néopolitique un Sarkozy en France est l'incarnation comme un Berlusconi en Italie ; et c'est cette néo-politique, brouillage des anciens clivages, qui aura par exemple permis à un Bertrand Delanoé, au moment de se déclarer candidat à la présidence du PS français, de s'afficher à la une du Nouvel Observateur, comme à la fois « Libéral et socialiste ». Dans le meilleur des mondes de la gouvernance et de la néo-politique, les clivages s'estompent, les significations se vident et les oxymores se multiplient, de social-libéral à libéral-social, signe parmi d'autres d'une efficace vidange des mots et des concepts.

Car cette langue de bois a aussi sa rhétorique et les opérateurs de cette rhétorique sont un autre aspect de nouveauté propre au langage du pouvoir aujourd'hui. Rhétorique de la métaphore, d'un côté, empruntant aux deux registres thématiques de l'économie et du sport de compétition, mais aussi à celui de la biologie dans l'esprit d'un darwinisme élargi à l'ensemble du monde social. Ainsi, la mondialisation, cet équivalent capitaliste de l'internationale communiste, est donnée pour une sorte de changement climatique planétaire, auquel nos sociétés seraient tenues de s'adapter ; ainsi, l'Europe ou le Marché, ces autres grandes entités transcendantes, exigeraient de nos sociétés transformation et mutation, tout se passant comme si la mondialisation était extérieure à toute décision politique et hors de prise, par conséquent, de toute politique, de la même manière que le Marché ou l'Europe sont donnés, dans le discours du pouvoir, comme des faits de nature, ou d'environnement, dit-on aussi. Il y a d'ailleurs quelque chose de curieux à remarquer que cette vision quasi écologique de l'économie mondialisée, sur laquelle les politiques n'auraient pas de prise et à laquelle il faudrait donc $s$ 'adapter, entre en contradiction - mais résolue en néopolitique - avec le volontarisme, l'initiative, la créativité qui font partie du fonds de commerce idéologique du libéralisme ; et cela aussi dans l'oubli que cette mondialisation des échanges, qu'on nous donne comme hors de portée de la décision politique, est pourtant bien orchestrée par des institutions ayant pignon sur la planète, du FMI à la Banque centrale.

Certains de ces nouveaux mots sont des sortes de monstruosités rhétoriques : ainsi flexisécurité (ouflexicurité) qui joue à la fois du mot valise et de l'oxymore. Mais en règle générale il est plus significatif que la plupart de ces mots relèvent d'une rhétorique de l'euphémisme, procédant soit par atténuation (restructurations, rationalisations, flexibilité), soit par formation pseudosavante (gouvernance, le politique, sociétal). Et ils relèvent bien en effet, d'un côté, d'une euphémisation du réel : derrière flexibilité, c'est la précarité qui se dissimule ; derrière rationalisations ou restructurations, ce sont des plans de licenciements, avec le cortège de difficultés, de drames et d'effets sociaux à long terme qui se dessinent. D'un autre côté, ces mots exercent donc aussi une très grande violence symbolique. Ils accompagnent et légitiment les violences qu'ils voilent pudiquement derrière le lexique de l'expertise, de l'efficacité, de la modernité. Qu'appelle-t-on aujourd'hui réformes (réformes évidemment nécessaires et dont il faut faire la 
pédagogie), sinon une politique de régression sociale, qui consiste en réalité à réformer les réformes du passé ? La romancière Annie Ernaux attribue très justement à ce lexique - dont elle a enregistré la montée en force dans sa magnifique autobiographie collective - un pouvoir de déréalisation. ${ }^{13}$ Le principe de réalité sociale ne s'en rappellera pas moins tôt ou tard à notre souvenir au-delà des lissages techno-économiques. Et ceux qui en paieront la note ne sont pas ceux qui, dans les sphères du pouvoir, nous paient actuellement de mots.

C'est dire, pour conclure, que la construction de la réalité politique et sociale dont ces « nouveaux mots du pouvoir » sont porteurs n'est pas seulement injuste ; elle est assez dangereuse. D'abord, en ce qu'elle est grosse d'un double processus de régression : régression sociale, dont les réformes, dans leur acception nouvelle, sont grosses, puisqu'il s'agit, au fond, de remonter, au nom de la modernité et du progrès, en amont des conquêtes sociales (qu'on aime, aujourd'hui, à appeler des acquis sociaux, dans l'oubli bien commode qu'il s'est agi de conquis sociaux); mais régression aussi idéologique, remettant à l'ordre du jour de la pensée dominante maints clichés du discours réactionnaire qui, au XIX ${ }^{\mathrm{e}}$ siècle, avait précisément cherché à faire bloc contre ce mouvement de conquête sociale.

Cette construction de la réalité politique et sociale peut paraître dangereuse, ensuite, en ce qu'elle vide de sens la démocratie, en ce qu'elle fait miroiter comme un horizon désirable une démocratie pacifiée, réduite à la paisible alternance au pouvoir de deux formations politiques dont l'une - la droite-énoncerait plus ou moins brutalement comme projet ce que l'autre - la gauche - ferait passer comme une solution. ${ }^{14}$ Une gauche qui pourrait être pensée comme l'édulcorant bien propre à faire avaler au plus grand nombre la tasse amère du capitalisme sauvage. Une gauche qui n'aurait plus guère avec la droite que quelques différences marginales, une façon de faire, une façon de dire, mais de faire et de dire en gros la même chose. Une gauche éthique et cosmétique, doucement libérale et gentiment libertine, conforme au modèle prémonitoire incarné par un journal comme Libération dans les années 80-90: favorable à la fois au libre-échange économique et au libre-échangisme sexuel.

Le danger réside enfin en ce qu'une démocratie ainsi lissée et dédialectisée stimule de façon diffuse un désenchantement politique dont elle se conforte en retour et pourrait ouvrir un boulevard aux extrémistes ou à ceux qui parviennent à concilier les extrêmes : dès lors que gauche et droite ne se distinguent plus guère que par des détails de procédure ou des degrés de radicalité dans un même projet, dès lors qu'on peut être à la fois « socialiste et libéral », le risque est grand de voir se répandre en Europe le modèle actuellement incarné par Nicolas Sarkozy et Silvio Berlusconi - ne serait-ce que parce que ceux qui pourraient faire barrage à un tel modèle auraient d'ores et déjà rendu les armes en troquant les convictions qu'ils sont censés défendre contre les mots et les valeurs qu'ils sont censés combattre. 
$\mathrm{N} \cdot \mathrm{O} \cdot \mathrm{T} \cdot \mathrm{E} \cdot \mathrm{S}$

1. Remy de Gourmont, Promenades philosophiques, première série (1905), dans La Culture des idées, éd. Dantzig, Paris, Laffont, coll. « Bouquins », 2008, p. 613.

2. Julien Green, L'avenir n'est à personne, Journal 1990-1992, Paris, Fayard, 1993, p. 73.

3. Pour Contrat Première Embauche, contrat d'emploi à durée indéterminée (mais avec périodes d'essai et de consolidation permettant à l'employeur de licencier sans motif) destiné aux moins de 26 ans et porté, sous le second mandat présidentiel de Jacques Chirac, par le premier ministre Dominique de Villepin. Publié au journal officiel le 2 avril 2006, le texte en sera abrogé le 21 avril, suite au mouvement social qu'il avait déclenché.

4. On ne nommera pas cet auteur : c'est qu'il s'agit non seulement d'une personnalité par ailleurs tout à fait respectable, mais surtout que son propos, uniment doxique, bien que signé, n'a rien au fond de référable à un individu singulier.

5. La campagne pour le référendum de 2005 sur le Traité constitutionnel européen a été, à cet égard, un moment particulièrement analyseur d'une telle représentation et surtout ce qui a suivi dans le discours des médias et des politiques (comme on le voit avec l'expression échec du référendum, qui s'est imposée comme naturellement pour désigner le fait que le vote n'ait pas conféré l'onction démocratique voulue aux orientations de ceux qui, au sommet de l'État, ont fait appel à lui). Quant à la ratification à la sauvette d'un « Traité » dit « simplifié », au mépris des engagements pris, elle en dit encore plus long à ce sujet. Les réactions politiques et médiatiques au résultat négatif du premier référendum irlandais sur le Traité de Lisbonne (2008) et l'organisation d'un second référendum un an plus tard ont abondé dans le même sens : celui d'un dévoilement des enjeux réels du scrutin (constitutionnaliser l'économie de marché) et du mépris dans lequel la classe dirigeante tient non seulement les peuples récalcitrants, mais encore les principes démocratiques dont elle persiste à se réclamer.

6. Voir sur ce point l'ouvrage désormais classique d'Albert O. Hirschman, Deux siècles de rhétorique réactionnaire, Paris, Fayard, 1991.

7. Pascal Durand (sous la dir. de), Les Nouveaux Mots du Pouvoir. Abécédaire critique, Bruxelles, Aden, 2007. Cet ouvrage a reçu à Paris le prix du Pamphlet décerné par les éditions Anabet. Voir aussi le dossier « Nouveaux Mots du Pouvoir. Fragments d'un abécédaire » (P. Durand dir.), dans Quaderni, $\mathrm{n}^{\circ} 63$, printemps 2007, 96 pages.

8. Pour Centre Démocrate Humaniste, nouvelle appellation du PSC (Parti Social-Chrétien).

9. L'expression est de Patricia Palermini (ULg), qui prépare à ce sujet un article de fond.

10. Guy Hermet, L'hiver de la démocratie ou le nouveau régime, Paris, Armand Colin, 2007.

11. Hannah Arendt, Walter Benjamin (1892-1940) [1955], trad. A. Oppenheimer-Faure \& P. Lévy, Paris, Allia, 2007, p. 108.

12. Voir Pierre Musso, Le Sarkoberlusconisme, Paris, Éditions de l'Aube, 2008.

13. «L'anomie gagnait. La déréalisation du langage grandissait, comme un signe de distinction intellectuelle. Compétitivité, précarité, employabilité, flexibilité faisaient rage. On vivait dans des discours nettoyés. On les écoutait à peine, la télécommande avait raccourci la durée de l'ennui » (Annie Ernaux, Les Années, Paris, Gallimard, 2008, p. 182).

14. Il n'est pas inutile de garder à l'esprit que le gouvernement Jospin a davantage privatisé que le gouvernement Balladur ; ce n'est pas, certes, que 
Balladur souhaitait moins de privatisations ; c'est qu'une réforme engagée par la gauche au pouvoir met moins massivement les travailleurs dans la rue. 